\title{
European Venus Explorer (EVE): an in-situ mission to Venus
}

\author{
E. Chassefière • O. Korablev • T. Imamura • \\ K. H. Baines • C. F. Wilson • D. V. Titov • \\ K. L. Aplin • T. Balint • J. E. Blamont • \\ C. G. Cochrane • Cs. Ferencz • F. Ferri • \\ M. Gerasimov • J. J. Leitner • J. Lopez-Moreno • \\ B. Marty • M. Martynov • S. V. Pogrebenko • \\ A. Rodin - J. A. Whiteway • L. V. Zasova • \\ J. Michaud • R. Bertrand • J.-M. Charbonnier • \\ D. Carbonne $\cdot$ P. Raizonville $\cdot$ EVE team
}

Received: 16 November 2007 / Accepted: 27 March 2008 / Published online: 31 May 2008

(C) Springer Science + Business Media B.V. 2008

\begin{abstract}
The European Venus Explorer (EVE) mission was proposed to the European Space Agency in 2007, as an M-class mission under the Cosmic Vision Programme. Although it has not been chosen in the 2007 selection round for programmatic reasons, the EVE mission may serve as a useful reference point for future missions, so it is described here. It consists of one balloon platform floating at
\end{abstract}

\footnotetext{
E. Chassefière $(\bowtie)$

Service d'Aéronomie/IPSL, UPMC University. CNRS, UVSQ, Paris, France

e-mail: Eric.Chassefiere@aero.jussieu.fr

O. Korablev $\cdot$ M. Gerasimov $\cdot$ L. V. Zasova

Space Research Institute, Russian Academy of Sciences, Moscow, Russia

T. Imamura

Institute of Space and Astronautical Science, Japan Aerospace Exploration Agency, Chōfu, Japan

K. H. Baines • T. Balint

Jet Propulsion Laboratory, California Institute of Technology, Pasadena, CA, USA

C. F. Wilson

Oxford University, Oxford, UK

D. V. Titov

Max Planck Institute for Solar System Studies, Lindau, Germany
}

K. L. Aplin

Rutherford Appleton Laboratory, Harwell Science and Innovation Campus, Oxfordshire, UK

J. E. Blamont · J. Michaud - R. Bertrand - J.-M. Charbonnier $\cdot$ D. Carbonne $\cdot$ P. Raizonville

Centre National d'Etudes Spatiales, Paris, France 
an altitude of 50-60 km, one descent probe provided by Russia, and an orbiter with a polar orbit which will relay data from the balloon and descent probe, and perform science observations. The balloon type preferred for scientific goals is one which oscillates in altitude through the cloud deck. To achieve this flight profile, the balloon envelope contains a phase change fluid, which results in a flight profile which oscillates in height. The nominal balloon lifetime is 7 days - enough for one full circumnavigation of the planet. The descent probe's fall through the atmosphere takes $60 \mathrm{~min}$, followed by $30 \mathrm{~min}$ of operation on the surface. The key measurement objectives of EVE are: (1) in situ measurement from the balloon of noble gas abundances and stable isotope ratios, to study the record of the evolution of Venus; (2) in situ balloon-borne measurement of cloud particle and gas composition, and their spatial variation, to understand the complex cloud-level chemistry; (3) in situ measurements of environmental parameters and winds (from tracking of the balloon) for one rotation around the planet, to understand atmospheric dynamics and radiative balance in this crucial region. The portfolio of key measurements is complemented by the Russian descent probe, which enables the investigation of the deep atmosphere and surface.

Keywords Planets and satellites · Venus · Planets and satellites · Formation · Radiative transfer $\cdot$ Hydrodynamics $\cdot$ Balloons $\cdot$ Space vehicles $\cdot$ Instruments

\author{
C. G. Cochrane \\ Imperial College London, London, UK \\ Cs. Ferencz \\ Eötvös University, Budapest, Hungary \\ F. Ferri \\ University of Padova, Padua, Italy \\ J. J. Leitner \\ University of Vienna, Vienna, Austria \\ J. Lopez-Moreno \\ Instituto de Astrofísica de Andalucía, Andalusia, Spain \\ B. Marty \\ Centre de Recherches Pétrographiques et Géochimiques, Nancy, France
}

M. Martynov

Lavochkin Association, Moscow, Russia

S. V. Pogrebenko

Joint Institute for VLBI in Europe, Dwingeloo, The Netherlands

A. Rodin

Moscow Institute of Physics and Technology, Moscow, Russia

J. A. Whiteway

York University, Toronto, Canada 


\section{Introduction}

The first phase of Venus spacecraft exploration by the Venera, Pioneer Venus, and Vega missions (1962-1992) included flyby spacecraft and orbiters, descent probes and balloons. It established a basic description of the physical and chemical conditions prevailing in the atmosphere and at the surface of the planet. Radar mapping by the Venera-15, Venera-16 and Magellan orbiters, combined with earlier glimpses from landers, provided a major expansion of our knowledge of Venus' geology and geophysics.

These early missions provided an ever-more detailed snapshot of the hostile conditions present on Venus today. However, they offered few clues as to how these extreme conditions had arisen. Many mysteries persist to this day: What surfaceatmosphere interaction exists to maintain such a massive atmosphere, including possible volcanism? What chemical and physical processes are responsible for the enormous, planet-encircling cloud layer? How did this planet evolve such a powerful greenhouse effect? At what stage in its history did Venus lose its magnetic field (and possibly plate tectonics), and how did this affect the evolution of its climate? What role did volcanism play in shaping the Venusian surface and degassing to its early atmosphere? How has the interaction of this planet with the solar wind determined the evolution of the climate? How is it that the atmosphere rotates 50 times faster than the solid planet below?

Beyond the specific case of Venus, resolving these issues is of crucial importance in a comparative planetology context, notably for understanding the long-term climatic evolution processes on Earth. Comparison of the climate on the three terrestrial planets would also help to determine the boundaries of the habitability zone in the Solar System, i.e., the range of distances to the Sun within which conditions on the planets can remain suitable for life.

After more than a decade of neglect, Venus is again being visited by a dedicated spacecraft. Venus Express (VEX), the first European Space Agency (ESA) spacecraft to Venus, was inserted into orbit around the planet on April 11, 2006. VEX focuses on the global remote sensing investigation of the atmosphere and plasma environment from a polar orbit [40]. Further down the road, in 2010, the Japanese space agency (Japan Aerospace Exploration Agency (JAXA)) plans their first mission to Venus, known as Planet-C or Venus Climate Orbiter, which will be dedicated to continuous meteorological observations of the planet [32]. Both VEX and Planet-C are remote sensing missions that provide a global survey of the atmospheric and plasma processes. The analysis of the data to be acquired by these missions will allow scientists to address many of the fundamental open issues mentioned earlier, but many of them will require further investigation with in situ measurements.

ESA's Venus Express mission provides a global survey of Venus, but there are many questions which cannot be addressed by remote sensing measurements alone, in particular those related to the isotopic ratios of noble gases and cloud chemistry cycles, issues which are the keys to understanding current climate and evolution of Venus and other terrestrial planets. We propose an in-situ mission, called the 
European Venus Explorer (EVE) and consisting of an orbiter, a balloon probe and a descent probe, to address the following science objectives:

1. To derive a unified model of the formation and evolution of terrestrial planets, by studying the record preserved in the atmospheric elemental and isotopic composition, together with data on escape processes.

2. To study the stability of the current climate on Venus, by quantifying exchange of atmospheric constituents with the surface and interior of the planet, and at the interface with space.

3. To study the complex chemical and radiative processes in the lower atmosphere and, in particular, in the cloud layer by in-situ measurements of gas and aerosol composition and radiative fluxes.

4. To re-construct the geological history of Venus, by mapping the subsurface structures by low frequency radar and to characterize the chemical composition of tesserae terrains.

5. To study the atmospheric dynamics, including the enigmatic super-rotation, using in situ probes and balloon trajectory tracking completed by remote sensing wind measurements.

6. To study electrical processes in the atmosphere through the search for lightning signatures by balloon and orbiter, and their potential impact on atmospheric electric and chemical properties.

The central theme of the mission is to understand the evolution of Venus and its climate, with relevance to terrestrial planets everywhere (including outside the Solar System).

\section{Science objectives}

The fundamental science issues that remain unfortunately unsolved by Venus Express and Planet-C remote surveys are the basis for formulating the science goals for the European Venus Explorer described in this section.

\subsection{Composition and chemistry}

The main components of the Venus atmosphere are $\mathrm{CO}_{2}(96.5 \%)$ and $\mathrm{N}_{2}(3.5 \%)$. Sulphur bearing gases, carbon and chlorine compounds, and water vapour are present in the atmosphere in amounts from few to few hundred parts per million [3, $21,44]$. The significant altitude and latitude variations of the abundances of the main trace gases imply that the minor constituents are involved in a number of chemical cycles and dynamical processes. Carbon monoxide is very abundant in the upper atmosphere due to photo-dissociation of $\mathrm{CO}_{2}$. The lower mesosphere $(60-70 \mathrm{~km})$ is a "photochemical factory" where the reactions between $\mathrm{CO}_{2}, \mathrm{SO}_{2}, \mathrm{H}_{2} \mathrm{O}$ and chlorine compounds induced by the solar ultraviolet (UV) radiation eventually lead to the formation of sulphuric acid aerosols. These processes result in strong $\mathrm{SO}_{2}$ and $\mathrm{H}_{2} \mathrm{O}$ gradients and significant temporal and spatial variability of their abundance at cloud tops.

The composition and chemistry of the lower atmosphere is poorly understood. It is thought that thermochemical cycles, involving sulphur and carbon species $\left(\mathrm{SO}_{2}\right.$, 
$\mathrm{CO}, \mathrm{COS}$ ) and water vapour, dominate in this region. The fast increase in COS abundance below the clouds results from the decomposition of sulphuric acid molecules. Surface minerals probably play a significant role in buffering the abundance of certain gases, such as $\mathrm{HCl}$ and $\mathrm{HF}$. The surprisingly low amount of molecular oxygen found in Venus' atmosphere may be attributed to binding of this gas by the minerals (e.g. iron oxides, carbonates, anhydrites), although escape may have played a role in removing oxygen $[11,12]$. Perhaps the most crucial question to resolve is what surface-atmosphere interaction occurs to maintain the massive $\mathrm{CO}_{2}$ atmosphere. A common theory involves buffering at the surface with carbonate minerals; however, it is unclear whether the concentration of $\mathrm{SO}_{2}$ in the deep atmosphere is too high to be in equilibrium with a carbonate surface $[4,21]$.

The composition of the mesosphere $(60-100 \mathrm{~km})$ is now being thoroughly investigated by Venus Express especially by solar occultation technique. However observations below the clouds are restricted to a few species and lack complete altitude coverage and sufficient vertical resolution. Among the primary objectives of the proposed mission is to measure in-situ the vertical profiles of the main trace gases $\left(\mathrm{SO}_{2}, \mathrm{CO}, \mathrm{COS}\right.$, $\mathrm{H}_{2} \mathrm{O}, \mathrm{O}_{2}, \mathrm{HCl}$ and others) in the troposphere from the cloud level to the surface and to investigate their latitude and local solar time variability within the clouds.

\subsection{Clouds and hazes}

Venus has the largest and the most complex cloud system among terrestrial planets. Clouds and hazes extend from 80 to $50 \mathrm{~km}$ with visible opacity in the range 20-40 and total mass loading varying from 1 to $10 \mathrm{mg} / \mathrm{m}^{3}$. Remote sensing observations showed that the upper cloud, at least, consists of micron-sized droplets of $75 \%$ $\mathrm{H}_{2} \mathrm{SO}_{4}$, which is photochemically produced at the cloud tops. Sulphuric acid was also detected by in-situ analysis on descent probes, but chlorine and phosphorous compounds were found to also constitute a significant portion of the lower cloud. An additional puzzle in the cloud composition is the nature of the unknown UV absorber that is responsible for the distinctive features seen at the cloud tops at the UV-blue wavelengths. Venus Express is currently monitoring morphology and global structure of the cloud layer but will not provide a definitive answer to the question of its chemical origin. The EVE mission will carry out an in-situ investigation of cloud particle composition. Together with the analysis of the gas phase these measurements will unveil the chemical cycles maintaining the giant cloud system.

Measurements by the Venera and Pioneer Venus descent probes sounded the vertical structure of the cloud as well as aerosol microphysical properties such as number density, size distribution, shape and refractive index [20]. One of the surprises was the discovery of a multi-modal size distribution that could indicate several distinct processes involved in the formation of the cloud system, and large particles $>3 \mu \mathrm{m}$ in size and number density $\times 10 \mathrm{~cm}^{-3}$ which are very probably solid crystals and thus cannot be composed of sulphuric acid. Recent observations suggest strong variability of the cloud properties all over the planet.

The proposed mission will significantly improve our knowledge of the Venus cloud system by in-situ characterization of the aerosol population and optical properties at different altitudes and locations. This data will be used to improve the optical model of the Venus clouds. 


\subsection{Origin and evolution}

Venus most likely received similar amounts of volatiles from the proto-planetary nebula as the Earth did. After the planets were formed various processes such as outgassing from interiors, escape from the top of the atmosphere, chemical interaction with the surface, and cometary impacts affected the volatile inventories. The role of these different processes in the evolution of terrestrial planets is poorly understood. Traces of these processes are recorded in the abundance patterns of noble gases and their isotopes. Measurements of the rare gases in the SNC meteorites from Mars, Earth mantle-derived rocks, and trapped in ices at $\sim 50 \mathrm{~K}$, suggest that: (1) the Earth and Mars formed their atmospheres mainly from interiors outgassing and cometary supply and (2) the Martian atmosphere was severely eroded by impacts. The pattern on Venus strongly differs, with greater relative amounts of neon and argon, placing the planet closer in this respect to meteorites and the Sun than the other terrestrial planets. Measurements of xenon and its isotopes on Venus which are currently lacking are of crucial importance for understanding the origin of the atmospheres of Venus and other terrestrial planets [17]. The European Venus Explorer will carry out in-situ measurements of the abundance of noble gases and their isotopes and especially those of xenon with high precision.

At present, there is relatively little water in any form on Venus, although it is not excluded that substantial amounts of water have been recycled to the mantle by past plate tectonics; but there are some indications that Venus may have had a watery past. These clues to the history of water are recorded in the value of the $\mathrm{D} / \mathrm{H}$ ratio, which was found to be $\times 150$ times higher in the lower atmosphere of Venus than on Earth. This enrichment can be explained by preferential escape of $\mathrm{H}$ atoms from the upper atmosphere and continuous supply of water to the atmosphere by comets or volcanism, or by a combination of both [26]. If there is little current influx of cometary or meteoritic water, this implies that a large amount of water has been lost from Venus, equivalent to a global ocean several tens of metres deep [18]. Recent Venus Express observations of $\mathrm{H}_{2} \mathrm{O}$ and $\mathrm{HDO}$ in the upper atmosphere have suggested about a factor of 2 higher $\mathrm{D} / \mathrm{H}$ ratio and its strong variability that can imply fractionation processes in the mesosphere [6], as observed in the atmosphere of Mars [5]. EVE will perform in-situ measurements of $\mathrm{H}_{2} \mathrm{O}$ and $\mathrm{HDO}$ abundance in the troposphere to determine boundary conditions for the $\mathrm{D} / \mathrm{H}$ fractionation in the mesosphere. This knowledge, combined with the surface composition data from ancient highlands, aims to resolve the controversy as to whether early-Venus was wet.

\subsection{Solar wind interaction and escape}

Tracing Venus' climatic evolution, requires a good understanding of the interaction of the atmosphere with the solar wind, as this governs the rates at which atmospheric constituents escape to space.

Since Venus has no internally generated magnetic field a large portion of its exosphere resides in the shocked solar wind flow and directly interacts with the solar wind. Photo-ionisation, charge exchange, electron impact processes and sputtering effectively remove exospheric components. Yet another loss mechanism occurs when the solar wind interacts with the top of the ionosphere to form a complex array 
of plasma clouds, tail rails, filaments and ionospheric holes on the night side through which a substantial amount of material leaves the planet.

Earlier missions studied these processes but the short lifetime of the Venera 9 and 10 orbiters, and insufficient temporal and mass resolution in the Pioneer Venus plasma instrument, did not allow a study of the mass and energy exchange between the solar wind and the upper atmosphere of Venus in sufficient detail. Venus Express continues investigation of the plasma environment by measuring the fluxes and energies of electrons, ions and energetic neutrals with simultaneous measurements of the magnetic field. Venus Express observations reveal the process of escape of $\mathrm{H}^{+}$, $\mathrm{He}^{+}$, and $\mathrm{O}^{+}$ions. Nevertheless, Venus Express is not equipped with low energy neutral and ion mass spectrometers able to directly measure neutral escape fluxes powered by sputtering and thermal mechanisms, and the low energy tail of pick-up ion escape flux distribution, where most of escape occurs.

Indeed, escape of neutral oxygen by sputtering is expected to be, together with plasma instabilities at the ionopause and pick-up $\mathrm{O}^{+}$escape, a major form of escape at Venus. The neutral escape flux of $\mathrm{O}$ has been estimated through modeling [17], but is still uncertain by two orders of magnitude. The neutral escape flux of hydrogen is similarly poorly known. Accurate observation of the $\mathrm{H}$ and $\mathrm{O}$ escape from the atmosphere is essential to trace back the climatic evolution of Venus. In a different perspective, the measurement of the ratio of $\mathrm{H}$ escape flux to $\mathrm{O}$ escape flux, and its comparison to the stoichiometric ratio $(2: 1)$, is fundamental to assess the role of surface rocks in removing (or not) oxygen from the atmosphere, i.e. the oxidant capacity of surface material. Understanding the detailed budget of helium sources and sinks is similarly of high interest to better understand the history of outgassing. The in-situ measurement by orbital mass spectrometry of the thermospheric-exospheric composition and neutral and ion escape fluxes, and of their responses to solar activity, will be done by EVE.

\subsection{Structure, dynamics and radiative balance}

The Venusian atmosphere has two global dynamical regimes: zonal super-rotation in the troposphere and mesosphere $(0-100 \mathrm{~km})$ [25] and solar-antisolar circulation across the terminator in the thermosphere (100-200 km) [9]. The observations show that the lower atmosphere has zonal wind velocities with maximum of $\sim 100 \mathrm{~m} / \mathrm{s}$ at the cloud tops decreasing to 0 at the surface and at the mesopause $(\sim 100 \mathrm{~km})$. In the mesosphere $(60-100 \mathrm{~km})$ the wind field is assumed to be related to the temperature structure by cyclostrophic balance. In addition to zonal super-rotation of the upper troposphere there appears to be a slower overturning of the atmosphere from equator to pole with meridional velocities of $3-10 \mathrm{~m} / \mathrm{s}$ [10] and giant vortices at each pole recycling the air downwards. Physical mechanisms maintaining the observed circulation pattern on Venus are virtually unknown, including the detailed structure of the thermal tides. Venus Express has started monitoring of the global dynamics. Planet-C plans to continue meteorological investigations. However both remote sensing missions are not capable of addressing crucial issues of heat and momentum transport in the lower atmosphere, which can be considered as the driving mechanism behind the super-rotation. EVE will complement the global monitoring from orbit by in-situ measurements of horizontal and vertical wind speeds and their 
fluctuations within the clouds where most of the solar energy is deposited. These investigations on the balloon carried around the planet by zonal circulation will cover all local times and significant latitude range, and will enable characterization of the thermal tides which may be key to driving the super-rotation. The descent probe will measure vertical temperature structure accurately enough to detect deviations from the adiabatic lapse rate and quantify convective stability.

The Venus mesosphere $(60-100 \mathrm{~km})$ is characterized by strong variability of the temperature and wind fields. A transition between two circulation regimes occurs at mesopause $(\sim 100 \mathrm{~km})$ where no wind measurements are available. The EVE orbiter will remotely sound this region with emphasis on direct wind velocity measurements. In addition, the orbiter will perform temperature and composition sounding in the mesosphere to recover the science goals of the unsuccessful planetary Fourier spectrometer (PFS)/Venus Express experiment.

The distribution of radiative fluxes in the dense Venus atmosphere determines the energy deposition pattern that eventually forces atmospheric motions. Only a few measurements of scattered solar radiation are available from the earlier descent probes. The EVE descent probe and balloon will perform measurements of the vertical profiles of scattered solar and thermal radiation. These observations together with the measurements of cloud structure and composition eventually aim at characterization of the radiative balance and its effect on the forcing of the atmospheric dynamics

One of the greatest uncertainties regarding climate change on Earth is the role of clouds. The clouds on Venus are known to play a critical role in maintaining its enormous greenhouse effect. The combination of measurements of the radiative balance, cloud properties, and dynamics will aim to clarify the complex coupled phenomena governing Venus' enormous greenhouse effect and assess its stability.

\subsection{Electrical environment}

There have been no in situ electrical observations at Venus, and the detailed nature of any discharges is still poorly understood. High temperature chemistry in the plasma in a lightning bolt can lead to the formation of trace species like NO, which may play an important role in atmospheric chemistry. The combination of in situ measurements of electrical, physical and chemical atmospheric properties with an orbiter for remote sensing is a powerful and unique combination. It will constrain cloud microphysics and contribute to the study of Venus' climate and radiative balance, in addition to providing detailed corroboration of existing observations.

There has been much debate over the existence of lightning on Venus [16]. Radio observations interpreted as lightning [34, 38] have not been widely corroborated optically, though some optical observations do exist [28]. NO detected in Venus' atmosphere can apparently only be produced by lightning [33]. More recent Galileo and Cassini radio observations suggested that Venus lightning is unlike terrestrial cloud-to-ground and intracloud lightning, and may resemble upper-atmosphere discharges such as sprites [27]. Although the breakdown potential in Venus' lower atmosphere is too high for cloud-to-ground lightning, the possible existence of cloud-to-cloud discharges in higher atmospheric regions has high scientific interest. On Earth, intracloud lightning frequently produces gamma ray flashes (e.g. [45]), 
and lightning-induced gamma ray bursts are also predicted in other planetary atmospheres [19]. Investigation of electromagnetic (EM) parameters (signal shape, spectral characteristic) and the occurrence density of both EM and gamma-ray events will assist understanding of the nature of the detected phenomena, its source altitude and generation mechanisms [23]. Observations of Venus lightning can contribute to the scientific understanding of convection and atmospheric chemistry, too. Lightning detected close to the surface could provide evidence for volcanism as the only charge generator powerful enough to act at these pressures. The general solid body activity of the planet, if it exists, can produce noise-like electric/EM events which can be detected as fast changes in the general electric/EM background. If natural extremely low frequency resonances ("Schumann resonances") appear in the surface-ionosphere waveguide, it indicates the global lightning activity of the planet independently from the detailed distribution and characteristics of lightnings. The EVE balloon will carry optical and acoustic lightning detectors as well as a more general electromagnetic event detector. The orbiter will carry an electromagnetic instrumentation suite comprised of an electromagnetic wave analyser, a camera to identify upper atmosphere discharges and a gamma-ray detector. As well as characterisation of the general electric/electro-magnetic environment at the orbiter, this unique instrumentation will permit synchronous detection of atmospheric electrical events using several methods.

Ionization by cosmic rays, which can penetrate to the surface of Venus, is a lightning-independent source of atmospheric charge. The presence of charged molecular cluster-ions and aerosols will produce atmospheric electric fields and a finite air conductivity [8, 41]. The properties of ions and aerosols are intimately linked and therefore measurements of non-lightning atmospheric electrical processes on Venus will contribute to understanding of trace gas chemistry, transport processes, and even cloud formation [1]. The EVE balloon will carry a permittivity instrument, comprising conductivity, electric field and electromagnetic wave detectors from which lightning, ion and aerosol properties can be determined.

The whole electric/electromagnetic environment of the planet contains the effects of the ionosphere-solar wind interactions. Such signals can be discriminated from signals originating from the lower atmosphere or from volcanism or seismic events. The EVE mission has the complete and unique capabilities to investigate the ionosphere-solar wind interaction, electrical effects and particle processes simultaneously in both the lower and upper atmosphere.

\subsection{Surface and interiors}

The radar images of the surface delivered by the Pioneer Venus, Venera-15, Venera16 , and Magellan orbiters surprisingly revealed that Venus possesses a young surface suggesting a geologically active past. Volcanism and tectonics have strongly altered the Venusian surface $[15,29]$ forming highly deformed old plateaus (Tesserae) and extensive lowlands (planitiae) — vast young volcanic plains covering about $80 \%$ of the surface. Relatively rare and uniformly distributed impact craters suggest that global resurfacing of Venus happened about 700 My ago $[2,36]$. The radar sounding experiment on board EVE orbiter will penetrate through the uppermost few hundred meters by means of wide-band low-frequency polarimetric radar sounding. This will 
unveil the terrains hidden under the young lava flows and potentially pyroclastics in an attempt to map subsurface stratigraphy, layering in multi-eruptive terrains, faults in potential subduction zones, and cooling joints-faults in volcanic tables (Heggy et al. 2006). This will shed light on the resurfacing history and the role of volcanism into gas transport to the atmosphere. The EVE radar will also explore the growth and evolution of large volcanic structures (individual volcanic edifices and coronae, and broad rises) that are key to understanding the geologic, magmatic, and thermal history of the solid part of Venus. Additionally, radar wide-band sounding will allow surface dielectric spectroscopy of the Venus surface to explore the upper tens of meters bulk mineralogical composition in smooth terrains.

Gravity anomalies were first measured by the Pioneer Venus Orbiter from 1978 to 1982, but the best gravity data were provided by the Magellan spacecraft from 1992 to 1994 . It will be possible, through accurate EVE orbitography using the $\mathrm{X} / \mathrm{Ka}$ transponder, to improve the $k_{2}$ coefficient and to investigate the properties of the Venusian crust and lithosphere below the observed targets. The variable part of the gravity field will provide information about the solid-planet tides and the atmosphere dynamics. If EVE could reduce by at least a factor of 5 the uncertainty on $\mathrm{k}_{2}$, it would not only allow the detection of whether the core is liquid, but would also provide a useful constraint on mantle properties [46].

The composition and mineralogy of the surface is to a great extent unknown. The Venera and Vega landers provided several geochemical measurements mainly consistent with basalts. However, all these samples were taken in the lowlands, so the highland tesserae regions, believed to represent the oldest terrain on Venus, still remain terra incognita. Therefore, the EVE descent probe will be targeted at one of these tesserae regions. The favoured landing site, Ovda Regio, rises $\sim 4 \mathrm{~km}$ above the surrounding plains and comprises a complex terrain with several generations of different structures [35]. The EVE mission will for the first time measure the surface composition in the tesserae regions, and will carry out panoramic imaging of the landing site. These measurements will contribute strongly to our understanding of Venus' mineralogical composition, its surface origin and volatile history.

Venus' surface and interior seem to play an important role in buffering current conditions on the planet and in the evolution of the climate. More than 50 years ago Urey [42] suggested that the current high surface pressure is maintained by the "carbonate buffer" - carbonate-silicate reactions on the surface [22]. However, the existence of carbonates on the Venus surface would result in conversion of the atmospheric $\mathrm{SO}_{2}$ into sulphates within a geologically short time of $\sim 2 \mathrm{My}$. Thus, the currently observed large abundance of $\mathrm{SO}_{2}$ is in strong disequilibrium with surface carbonates and could indicate ongoing volcanic activity that replaces sulphur dioxide removed by reaction with the minerals. Hashimoto and Abe [30] considered an alternative "pyrite buffer" that is in equilibrium with the observed amount of atmospheric $\mathrm{SO}_{2}$. However the stability of pyrite under Venus conditions is also an open issue. The surface could be a buffer for atmospheric gases like $\mathrm{HCl}$ and $\mathrm{HF}$ as well as taking up oxygen released in the photo-dissociation of water vapour and carbon dioxide in the upper atmosphere. One of the main goals of the EVE lander is to provide in-situ investigation of the surface composition in order to determine the buffering effect on the current atmospheric chemistry and thereby enabling a decision between the carbonate and pyrite buffer models. 


\subsection{Exobiology}

Given the priority specified in ESA's Cosmic Vision programme to the theme of "Life and habitability", it is important to consider how the EVE mission can help to address this topic.

To understand the emergence of life, it is clearly important to measure throughout our solar system the inventories of the building blocks of life, ranging from water and oxygen through to more complex carbon-containing compounds found for example in carbonaceous meteorites. The EVE mission will quantify the abundances of elements important for life, such as carbon, oxygen, nitrogen and iron, and will also have the capability of identifying more complex carbon-based compounds should any be present in the clouds.

Also important from an astrobiological perspective is to determine (1) the flux of meteorites and micro-meteorites entering the atmosphere of Venus, and (2) the fate of carbon-containing materials carried by meteorites. EVE will carry a high-speed camera which, in addition to measuring lightning, will quantify the meteor flux at the top of the Venus atmosphere. As to the second objective, we do not expect to find measurable quantities of carbon-bearing compounds in the clouds of Venus; however, the chemical characterisation of the cloud layer provided by the balloon instruments will enable us to model the fate of meteoritic material in this environment.

The clouds of Venus are particularly interesting in that they represent one of the very few places in our solar system in which liquid water is found, albeit in the form of concentrated sulphuric acid with a $\mathrm{pH}$ of roughly zero. These conditions are not as far from habitability as might at first be thought: Extremophiles have been found on Earth living in conditions of $\mathrm{pH} 0$ [39], and other bacteria have been found living in cloud droplets (e.g. [24]). Although it is extremely unlikely to find life in the combination of high acidity and low nutrient availability found in the clouds of Venus [14], it is still of biochemical interest to study whether complex carbon compounds can be found here, whether of indigenous origin or carried by meteorites. This is especially of interest given the parallels between this environment and that of Earth in its first few tens of millions of years, when it exhibited a mainly $\mathrm{CO}_{2}$ atmosphere (see e.g. [47]).

The possibility that Venus once had vast oceans of water is clearly of great exobiological interest (see e.g. [43]). As discussed above, the EVE mission will search for evidence of this hypothesis by measuring isotopic and elemental abundances.

\section{Mission baseline}

EVE is an "M-class" mission-roughly equivalent to NASA's "Discovery class" missions - which means that the total cost to ESA is estimated to be $300 \mathrm{M} €$. This figure does not include the cost of scientific instruments, which would be funded by individual nations, nor does it include sizable international contributions to the mission, in particular from Russia. This relatively low cost for such an ambitious mission architecture is made possible by taking advantage of the extensive heritage 
from the Russian Venera and Vega entry probes, and in particular from the FrenchRussian collaboration on Vega balloons.

EVE consists of one balloon platform floating at an altitude of 50-60 km, one descent probe provided by Russia, and an orbiter with a polar orbit which will relay data from the balloon and descent probe, and perform science observations. Several kinds of balloon have been proposed for planetary exploration [7]. The balloon type preferred for EVE scientific goals is one which allows repeated vertical excursions through the cloud deck. A suitable flight profile will be obtained by filling the balloon envelope with a phase change fluid such as water, resulting in a flight profile which oscillates in altitude [13, 37]. However, most of the science goals can also be achieved using a helium superpressure balloon, as previously used for the Vega balloon missions, which would maintain a near-constant altitude for the duration of the mission. The nominal balloon lifetime is 7 days - enough for one full circumnavigation of the planet. The descent of the probe through the atmosphere takes $60 \mathrm{~min}$, followed by $30 \mathrm{~min}$ of operations at the surface. The nominal orbiter life time is 2 years.

The EVE spacecraft is launched via Ground Transfer Orbit (GTO) by a Soyuz Fregat $2-1 b$ from Kourou. The spacecraft delivers the balloon and descent probes to Venus from transfer orbit, and then is itself inserted into orbit around Venus. The orbiter performs data relay from the descent probe and balloon, while also performing context science observations. After the end of balloon operations, the orbit is lowered using aero-braking, to optimise further science investigations. The balloon and the descent probe are both tracked by Earth-based very long base interferometry (VLBI) to derive winds in the lower atmosphere.

The EVE balloon carries comprehensive chemistry and isotopic analysers, focussing on cloud-level processes. The key instrument is a state of the art gas chromatograph/ mass spectrometer (GCMS) system to analyse cloud and gas composition. Other instruments provide optical investigations of aerosol composition, microphysical properties, and radiative balance. In particular, the balloon provides a stable platform for the long integration times ( hours) required for isotopic mass spectrometry. The feasibility of deploying and operating balloons on Venus was demonstrated in 1984 by the Vega balloons-but those balloons carried only a very small payload of pressure, temperature, light flux, and backscatter sensors, and operated for only $48 \mathrm{~h}$ compared to the 7 day minimum lifetime specified for the EVE balloon.

The orbiter carries a range of instruments to complement the in situ measurements of the probes. A low-frequency radar for subsurface sounding and a sub-millimetre instrument to directly measure atmospheric winds will be used at Venus for the first time. A thermal infrared (IR) spectrometer will recover the science goals of the nonoperational PFS instrument on Venus Express.

The Russian descent probe carries instruments similar to the balloon payload, including a GCMS, but will focus on the vertical profiles of isotopic and molecular abundances, aerosol properties, radiative fluxes and convective stability. A particular scientific objective is the characterization of near-surface chemistry. The probe will return images during descent and from the surface, and measure surface composition after landing e.g. by gamma-ray spectroscopy. The planned landing site is in the highland tesserae regions, which are understood to be the oldest terrain on Venus, and have not yet been visited by any spacecraft. 
The Japanese space agency (JAXA) may provide a small, water vapour-inflated balloon which would be deployed at $35 \mathrm{~km}$ altitude and would communicate directly with Earth. This balloon would carry a few meteorological sensors and a radio beacon for trajectory determination, with a goal of determining the circulation at this altitude, which is important for understanding the atmospheric super-rotation. The JAXA balloon is an optional element of this mission.

The entry mass of the balloon and descent probes is $170 \mathrm{~kg}$ each, and the orbiter dry mass is $690 \mathrm{~kg}$ before adding system margins; once $1800 \mathrm{~kg}$ of fuel have been added as well as various system margins, the total launch mass is roughly $3,000 \mathrm{~kg}$, consistent with a Soyuz-Fregat 2-1b launch via GTO.

The mass of the science payload, including a $20 \%$ margin, is $52 \mathrm{~kg}$ for the orbiter, $12 \mathrm{~kg}$ for the balloon and $24 \mathrm{~kg}$ for the descent probe.

\section{Scientific payload}

\subsection{Orbiter model payload}

The goals of the orbiter are to provide data relay from the descent probe and balloon, to provide remote sensing context for the balloon and descent probe, and to perform further remote sensing investigations after the balloon and descent probes have completed their missions. The scientific payload is summarized below:

Neutral mass and energy spectrometer This instrument will measure the density (above a few tens per cubic centimetre) and the mass and energy distribution of the low energy neutral particles at the orbit in order to quantify and map the neutral escape fluxes.

Panoramic ion mass-spectrometer The mass composition (1-100 amu), density, velocity and temperature of super-thermal and hot ions will be measured in the energy range from few electron volts to $10 \mathrm{keV}$ to characterize ion escape fluxes.

Ionospheric plasma UV imager An ultraviolet imager, provided by Japan, will map the EUV emission resonantly scattered by $\mathrm{O}$ and $\mathrm{He}$ ions in the ionosphere in such a way to characterize ionospheric convection patterns and solar wind-ionosphere interaction.

Sub-millimetre sounder A devoted microwave spectrometer will provide the mesospheric wind field and investigate with an unprecedented accuracy the atmospheric composition $\left(\mathrm{H}_{2} \mathrm{O}, \mathrm{CO}, \mathrm{ClO}, \mathrm{BrO}, \mathrm{SO}, \mathrm{SO}_{2}, \mathrm{H}_{2} \mathrm{~S}, \mathrm{NO}, \mathrm{HO}_{2}, \mathrm{H}_{2} \mathrm{O}_{2}\right.$, $\mathrm{HCl}, \ldots$ ), temperature field and heat flux between $\approx 60$ and $\approx 140 \mathrm{~km}$.

Ultraviolet mapping spectrometer This instrument, working in limb and nadir viewing modes, will investigate the detailed ultraviolet spectral characteristics of Venus atmosphere to retrieve structural, dynamical and composition data, to analyze cloud composition (in particular search for the nature of the "unknown UV absorber"), and to search for night side airglows. 
Lidar A lidar, provided by Canada, will measure the vertical profiles of the atmospheric backscattering ratio and, by using two different wavelengths, will return information about particle size and type. This instrument will provide an accurate altimetry of the top of the main cloud layer and the vertical structure of overlying hazes to better characterize atmospheric dynamics, convection and cloud processes.

Infrared Fourier spectrometer This spectrometer, recovering the science of the nonoperational PFS instrument on Venus Express, will measure the three-dimensional temperature fields on the day and night sides and will provide the composition of the atmosphere at 60-100 km, synergistically with the microwave spectrometer.

High-speed camera A high speed camera will be used to provide context imaging and to track clouds, in view to complement wind measurements made by the microwave sounder from the orbiter and from VLBI tracking of the balloon(s). The camera will be used also to detect and quantify luminous phenomena like lightning and meteor falls.

Radio interferometry and Doppler tracking Basic atmospheric and gravity data will be derived by the combined use of VLBI and accurate Doppler tracking through the use of a transponder onboard the orbiter.

Electromagnetic wave analyzer This instrument will provide a continuous monitoring of the electromagnetic background and discrete events, simultaneously with measurements made from the balloon and the descent probe.

Subsurface and ionospheric radar The radar has been specifically designed to probe volcanic terrains. During the low-altitude part of the orbit, it will probe the subsurface down approximately to $300 \mathrm{~m}$ depth with $2-5 \mathrm{~m}$ vertical resolution in order to map structural anomalies. It will be also able to probe from the high-altitude part of the orbit possible ionospheric fluctuations in response to quakes, providing information on seismic activity and internal structure.

DC magnetometer This precise three-axial (vector) flux-gate magnetometer will map the magnetic field, with the main goal to help in the interpretation of particle measurements in view to characterize atmospheric escape.

Gamma flash detector Possible gamma-bursts produced by thunderstorms will be searched for and characterized, complementing visible and radio wave measurements of lightning activity from the orbiter and probes.

\subsection{Balloon model payload}

The EVE balloon will carry a scientific payload focused on cloud-level, long term (1 week) in-situ measurements.

The main science goals to be addressed by the balloon payload are (1) to investigate cloud-level processes, which includes: gas composition, cloud particle composition, cloud particle size and refractive index, radiative balance, vertical 
winds and turbulence, atmospheric electricity, (2) to provide a stable, longduration platform for measurement of the isotopic ratios of light elements and noble gases.

The following payload is chosen to address the above objectives:

GCMS A gas chromatograph/mass spectrometer combination, focused on atmospheric and aerosol composition. A dedicated aerosol collection inlet, with heritage from Huygens ACP, enables discrimination between gas and aerosol composition.

Isotopic MS A dedicated high-resolution mass spectrometer optimised for the study of isotopic ratios. It is equipped with cryotraps and chemical getters to remove $\mathrm{CO}_{2}$ and $\mathrm{N}_{2}$ - which together make up $99.9 \%$ of the atmosphere-from the gas sample before MS analysis.

Polarising nephelometer This instrument is included to study the cloud particles in detail, and will return the size distribution and refractive index of aerosol particles, as well as some indication of the shape. This instrument is critical to resolve current uncertainties about the nature of Venus cloud particles.

Optical package This light instrument will measure upward and downward radiation, in six spectral bands from UV to thermal IR, in order to characterise the radiative balance, and thus the greenhouse effect. This instrument will also have a lightning detection mode.

Meteorological package This package will include pressure and air temperature sensors, as well as a sound detector to characterise the acoustic environment including the search for thunder. An accelerometer to determine pressure and density profiles during entry will also be included.

VLBI S-band beacon with USO This is included in order to permit accurate tracking of the balloon from Earth using phase-referencing VLBI to measure the wind characteristics on different scales.

Electrical and electromagnetic properties package This instrument will include two electrical antennae, which can be used in several modes. It can be used in DC mode to measure electric field; in pulsed mode to measure electrical permittivity; and in $\mathrm{AC}$ mode to measure the EM spectrum and events at the balloon.

IR spectrometer An Attenuated total reflection (ATR) spectrometer will be used to obtain absorption spectra of the aerosols, in order to reduce ambiguity in chemical identification which may remain after the GCMS results. ATR spectrometry is particularly important for identifying pre-biotic or biotic constituents.

Data are transmitted to Earth through the orbiter communication system. The average data rate is 100 bits per second during the balloon mission, or a cumulated 70 Mbits of scientific data collected by the orbiter during 1 week balloon mission and transmitted to Earth. 


\subsection{Descent probe model payload}

The EVE descent probe measurements are dedicated to atmospheric profiling with focus on deep atmospheric layers, and to measurements of surface composition.

The following payload is chosen to address the following objectives:

Chemistry package A gas chromatograph/mass spectrometer combination, focused on atmospheric composition $\left(\mathrm{H}_{2} \mathrm{O}, \mathrm{CO}, \mathrm{CO}_{2}\right.$, sulphur compounds) with tuneable diode laser absorption spectrometer) aimed to measure the vertical profiles of the isotopes of $\mathrm{CO}_{2}$ and $\mathrm{H}_{2} \mathrm{O}$. This instrument will also perform measurements of noble gases (Ne, Ar, Kr, Xe). The composition of different clouds layers will be measured separately via pyrolysis techniques and an aerosol collection inlet. In the low atmosphere, an oxygen fugacity sensor will measure $\mathrm{O}_{2}$ content, and hightemperature gas sensors of silicon carbide will back-up measurements of $\mathrm{SO}_{2}, \mathrm{O}$, and $\mathrm{CO}$.

Optical package An instrument to measure spectrally resolved upward and downward radiation, from UV to $3.8 \mu \mathrm{m}$. UV-visible part will allow profiling of $\mathrm{SO}_{2}$ and SO. The Infrared part $(0.8-3.8 \mu \mathrm{m})$ is dedicated to the characterization of the radiative balance, and will also provide vertical profile of $\mathrm{H}_{2} \mathrm{O}$.

ATR spectrometer An infrared spectrometer for contact analysis of aerosol droplets. Attenuated total reflection spectra in a wide thermal-IR range will be obtained to characterize the chemical composition of cloud droplets. Twenty different prisms are included in the instrument, allowing the characterisation of at least 20 and perhaps up to 200 samples. The ATR spectrometer has a potential to detect amino acids, and other biological substances.

Nephelometer An optical instrument allowing characterization of optical properties of cloud particles, such as size distribution and refractive index by measuring diffused radiation at different angles and at different wavelengths. The resulting profile of cloud parameters is important to characterise the heat balance on Venus.

Meteorological package This package will include pressure and temperature sensors to work during descent, and an anemometer to measure horizontal wind while on the surface.

Accelerometer The acceleration profile in the upper atmosphere before parachute deployment will be used to derive the density profile of the atmosphere.

Imaging system A set of descent and panoramic cameras. The descent camera will image the landing site during descent in the $0.9-\mu \mathrm{m}$ transparency window to assess landing site geology, and to try to locate the landing site on Magellan maps. The colour panoramic stereo camera will be used to obtain the surface panorama. One descent camera and four panoramic cameras (one of them stereo) are foreseen resulting in six camera heads with common electronic block. 
Gamma-spectrometer with neutron activation This instrument performs comprehensive analysis of Venus soil elemental composition at the landing side with accurate determination of content of soil-composing elements $\mathrm{Si}, \mathrm{O}, \mathrm{Ca}, \mathrm{Na}, \mathrm{Al}, \mathrm{Mg}, \mathrm{Fe}$, Ti, etc. as well as of natural radioactive isotopes $\mathrm{K}$, Th and $\mathrm{U}$. The method of investigation is based on measurements of gamma-ray lines of radioactive isotopes and secondary neutrons and gamma-ray lines, which are produced by soil-composing nuclei in reactions with $14 \mathrm{MeV}$ neutrons from the Pulsing Neutron Generator. The gamma-ray spectrometer will be located inside the instrument container, and will be operated only while on the surface (integration time of $30 \mathrm{~min}$ ).

Alternatively to the Gamma-neutron spectrometer, a sampling system coupled with a laser-induced plasma mass-spectrometer would provide measurements of rock-forming and trace elements.

Lightning detector This instrument will include one electrical and one magnetic antenna, and a low-frequency $(8 \mathrm{kHz}-2 \mathrm{MHz}$ for magnetic component and DC $-50 \mathrm{kHz}$ for electric component) spectrum analyser to measure the EM spectrum and detect lightning events.

The data rate which can be transmitted from the probe to the orbiter is likely to be of the order of $1 \mathrm{kbit} / \mathrm{s}$, although this number is sensitive to orbital assumptions. During the $\sim 5,000 \mathrm{~s}$ lifetime of the descent probe, then, the expected data volume is expected to be of the order $\sim 5$ Mbits.

\subsection{Low altitude JAXA-provided balloon probe model payload}

Direct observations of the winds and temperature profile in the sub-cloud region of Venus are important for solving fundamental problems regarding Venus' environment. The small, low-altitude balloon provided by JAXA as an optional payload will give key information on the dynamics of the lower atmosphere. The balloon will float at $35 \mathrm{~km}$ altitude, which is more than one scale height lower than the cloud base, and circle around the planet thanks to the super-rotation of the atmosphere. This balloon will complement the meteorological observations by the cloud-level main balloon; simultaneous observations by the two balloons will characterize threedimensional atmospheric motions.

The balloon will carry an S-band transmitter to be continuously located by VLBI from the Earth. From the trajectory of the balloon, the structures of meridional circulation and synoptic-scale to planetary-scale waves will be constrained. The balloon will be also equipped with temperature and pressure sensors, and their data will be transmitted directly to the ground station on the Earth with a telemetry rate on the order of 1 bps. The analysis of balloon dynamics with temperature and pressure time series gives vertical motion and eddy heat flux, thereby constraining the static stability, thermal convection and gravity wave activity. Optional instruments include an irradiance monitor and a nephelometer to address cloud physics. The combination of these data, covering from meso-scale to planetary scale, gives clues to the mechanism of the super-rotation, the energy transfer and the material circulation in the lower atmosphere.

A table relating relevant Venus science themes to EVE instruments is given in Table 1 . 
Table 1 Science themes/instrument diagram

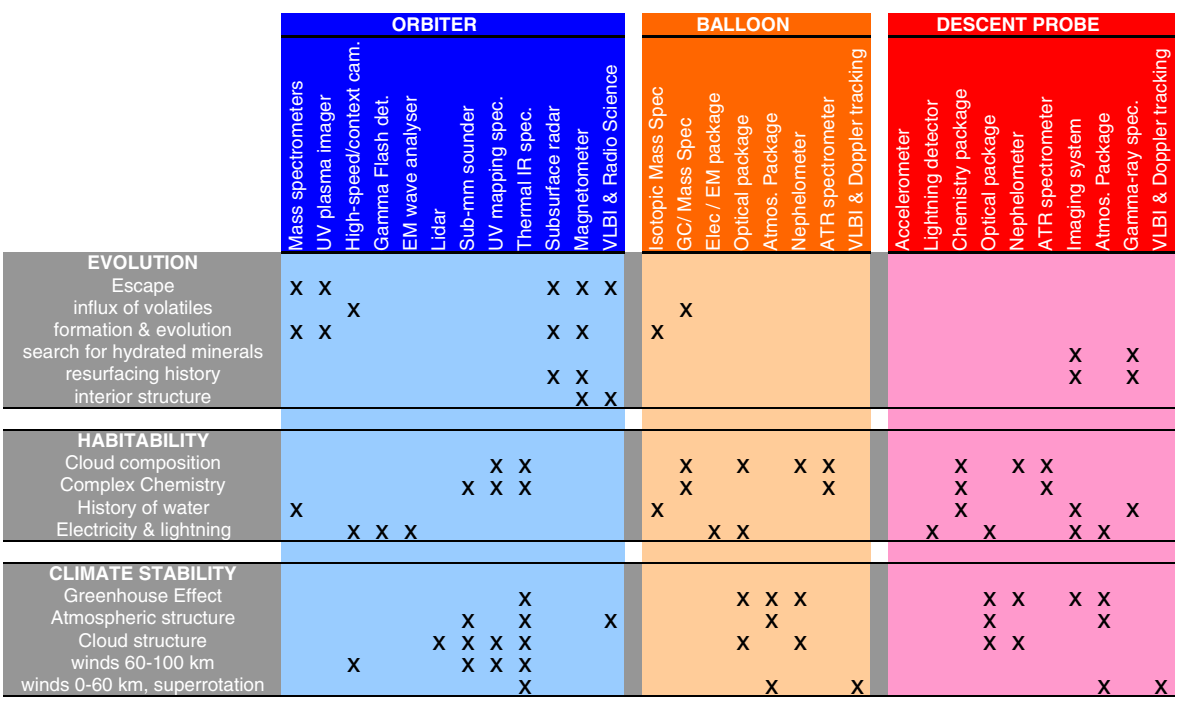

\section{Conclusion}

Although EVE has not been selected by ESA for further assessment study, it may be thought that it will open the way to a strong involvement of Europe in future Venus exploration. According to the recommendations of ESA working groups, key technologies related to the use of balloons for planetary exploration will be included in the technology development programmes of ESA, allowing to improve their degree of technological readiness.

Potential international partners are all developing ambitious plans for Venus insitu exploration, in the follow-up of the European Venus-Express and Japanese Venus Climate orbiter missions. The VENERA-D mission, which will mainly consist of a long-lived lander, is planned in the Russian federal programme and should be launched during the next decade. Following the recommendations of the American Venus Exploration Analysis Group, a New-Frontier-class in-situ mission might be flown by NASA at the middle of the next decade, and would be possibly used to validate key technologies for a further more ambitious Flagship mission in 2020-2025. JAXA is studying a low altitude balloon for probing the low atmosphere of Venus. This balloon could be flown to Venus, either on a small Japanese domestic mission, or in the frame of a multiplatform international EVE-type mission, in the next decade. Because of the difficult access to Venus lower atmosphere and surface, it clearly appears that understanding the evolution and climate of Venus is not achievable with a single mission by a single agency. A multi-agency, multi-mission coordinated approach is the key condition of a scientifically successful programme of Venus exploration, and EVE could be a major element of such an international programme. 


\section{References}

1. Aplin, K.L.: Surv. Geophys. 27, (1), 63 (2006)

2. Basilevsky, A.T., Head, J.W., Schaber, G.G., Strom, R.G.: The resurfacing history of Venus. In: Bougher, S.W., Hunten, D.M., Phillips, R.J. (eds.) Venus II, p. 1047. University of Arizona Press, Tucson (1997)

3. De Bergh, C., Moroz, V.I., Taylor, F.W., et al.: Planet. Space Sci. 54, 1389 (2006)

4. Bertaux, J.L., Widemann, T., Hauchecorne, A., et al.: J. Geophys. Res. 101, 12709 (1996)

5. Bertaux, J.L., Montmessin, F.: J. Geophys. Res. 106, 32879 (2001)

6. Bertaux, J.L., Vandaele, A.-C., Korablev, O., et al.: Nature 450, 646 (2007)

7. Blamont J.: In: Maran, S.P. (ed.) The Astronomy and Astrophysics Encyclopedia, p. 494. Cambridge University Press, Cambridge (1991)

8. Borucki, W.J., Levin, Z., Whitten, R.C., et al.: Icarus 51, 302 (1982)

9. Bougher, S.W., Alexander, M.J., Mayr, H.G.: Geology, geophysics, atmosphere, and solar wind environment. In: Bougher, S.W., Hunten, D.M., Phillips, R.J. (eds.) Venus II, p. 259. University of Arizona Press, Tucson (1997)

10. Carlson, R.W., Baines, K.H., Encrenaz, T., et al.: Science 253, 1541 (1991)

11. Chassefière, E.: J. Geophys. Res. 101, 26039 (1996)

12. Chassefière, E.: Icarus 126, 229 (1997)

13. diCicco, A.G., Nock, K.T., Powell, G.E.: AIAA 95, 1633 (1995)

14. Cockell, C.: Planet. Space Sci. 47, (12), 1487 (1999)

15. Crumpler, L.S., Aubele, J.C., Senske, D.A., et al.: Volcanism on Venus. In: Bougher, S.W., Hunten, D.M., Phillips, , , R.J. (eds.) Venus II, p. 697. University of Arizona Press, Tucson (1997)

16. Desch, S.J., Borucki, W.J., Russell, C.T., Bar-Nun, A.: Rep. Prog. Phys. 65, 955 (2002)

17. Donahue, T.M., Russell, C.T.: In: Bougher, S.W., Hunten, D.M., Phillips, R.J. (eds.) Venus II, p. 3. University of Arizona Press, Tucson (1997)

18. Donahue, T.M., Grinspoon, D.H., Hartle, R.E., Hodges Jr., R.R.: Ion neutral escape of hydrogen and deuterium: evolution of water. In: Bougher, S.W., Hunten, D.M., Phillips, R.J. (eds.) Venus II, p. 385. University of Arizona Press, Tucson (1997)

19. Dwyer, J.R., Coleman, L.M., Lopez, R., et al.: Geophys. Res. Lett. 33, L22813 (2006)

20. Esposito, L.W., Knollenberg, R.G., Marov, M.Y., Toon, R.B., Turko, R.P.: The clouds and hazes of Venus. In: Hunten, D.M., Colin, L., Donahue, T.M., Moroz, V.I. (eds.) Venus, p. 484. University of Arizona Press, Tucson (1983)

21. Esposito, L.W., Bertaux, J.-L., Krasnopolsky, V., Moroz, V.I., Zasova, L.V.: Chemistry of lower atmosphere and clouds. In: Bougher, S.W., Hunten, D.M., Phillips, R.J. (eds.) Venus II, p. 415. University of Arizona Press, Tucson (1997)

22. Fegley Jr., B., Klingelhöfer, G., Lodders, K., Widemann, T.: Geochemistry of surface-atmosphere interactions on Venus. In: Bougher, S.W., Hunten, D.M., Phillips, R.J. (eds.) Venus II, p. 591. University of Arizona Press, Tucson (1997)

23. Ferencz, C., Ferencz, O.E., Hamar, D., Lichtenberger, J. (eds.): In: Whistler phenomena, short impulse propagation, p. 170. Kluwer Academic, Dordrecht (2001)

24. Fuzzi, S., Mandrioli, P., Perfetto, A.: Atmos. Environ. 31, 287 (1997)

25. Gierasch, P.J., Goody, R.M., Young, R.E., et al.: Atmosphere and solar wind environment. In: Bougher, S.W., Hunten, D.M., Phillips, R.J. (eds.) Venus II, p. 459. University of Arizona Press, Tucson (1997)

26. Grinspoon, D.H.: Nature 363, 428 (1993)

27. Gurnett, D.A., Zarka, P., Manning, R., et al.: Nature 409, 313 (2001)

28. Hansell, S.A., Wells, W.K., Hunten, D.M.: Icarus 117, 345 (1995)

29. Hansen, V.L., Willis, J.J., Banerdt, W.B.: Tectonic overview and synthesis. In: Bougher, S.W., Hunten, D.M., Phillips, R.J. (eds.) Venus II, p. 797. University of Arizona Press, Tucson (1997)

30. Hashimoto, G., Abe, Y.: Planet. Space Sci. 53, 839 (2005)

31. Heggy, E., Clifford, S.M., Grimm, R.E., et al.: J. Geophys. Res. 111, 2589 (2006)

32. Nakamura, M., Imamura, T., Ueno, M., et al.: Planet. Space Sci. 55, (12), 1831 (2007)

33. Krasnopolsky, V.A.: Icarus 182, 80 (2006)

34. Ksanfomality, L.V., Scarf, F.L., Taylor, W.W.L.: In: Hunten, D.M., Colin, L., Donahue, T.M., Moroz, V.I. (eds.) Venus, p. 565. University of Arizona Press, Tucson (1983) 
35. Leitner, J.J., Firneis, M.G., Aittola, M., et al.: Geophys. Res. 9, Abstract (2007)

36. McKinnon, W.B., Zahnle, K.J., Ivanov, B.A., Melosh, H.J.: Catering on Venus: models and observations. In: Bougher, S.W., Hunten, D.M., Phillips, R.J. (eds.) Venus II, p. 969. University of Arizona Press, Tucson (1997)

37. Nock, K.T., Aaron, K.M., Jones, J.A., et al.: AIAA 95, 1632 (1995)

38. Russell, C.T.: Space Sci. Rev. 55, 317 (1991)

39. Schleper, C., Peuhler, G., Holz, I., Gambacorta, A., Janekovic, D., Santarius, U., Klenk, H.P., Zillig, W.: J. Bacteriol. 177, 7050 (1996)

40. Svedhem, H., Titov, D., McCoy, D., et al.: Planet. Space Sci. 55, (12), 1636 (2007)

41. Tzur, I., Levin, Z.: Icarus 52, 346 (1982)

42. Urey, H.C.: The Planets. Yale University Press, New Haven (1952)

43. Venus Exploration Analysis Group: Venus Exploration Goals, Objectives, Investigations, and Priorities, a report of the Venus Exploration Analysis Group (VEXAG). VEXAG, Washington, D.C. (2007) October

44. Von Zahn, U., Moroz, V.I.: Adv. Space Res. 5, 173 (1985)

45. Williams, E., Boldi, R., Bor, J., et al.: J. Geophys. Res. 111, D16209 (2006)

46. Yoder, C.F.: Venusian spin dynamics. In: Bougher, S.W., Hunten, D.M., Phillips, R.J. (eds.) Venus II, p. 1087. University of Arizona Press, Tucson (1997)

47. Zahnle, K., Arndt, N., Cockell, C., Halliday, A., Nisbet, E., Selsis, F., Sleep, N.: Space Sci. Rev. 129, (1-3), 35 (2007) Online First (SSRv Homepage) 